\title{
Recent developments in transsphenoidal surgery of pituitary tumors
}

\author{
Panagiotis Nomikos ${ }^{1}$, Rudolf Fahlbusch ${ }^{2}$, Michael Buchfelder ${ }^{1}$
}

\begin{abstract}
${ }^{1}$ Department of Neurosurgery, University of Goettingen, Goettingen, Germany and ${ }^{2}$ Department of Neurosurgery, University of Erlangen-Nuremberg, Erlangen, Germany
\end{abstract}

\section{INTRODUCTION}

Despite recent and ongoing advances in the pharmacological and radiotherapeutic management of pituitary tumors, surgery remains the therapy of choice for the vast majority of these lesions. Both transsphenoidal and transcranial microsurgery of pituitary adenomas and of other sella lesions are well established, safe and highly efficient therapeutic procedures. As surgical success is now being judged by more stringent endocrine criteria and more sophisticated imaging than ever before, advances in the surgical technique resulting from the ongoing technical evolution have been eagerly awaited. Despite the fact that transsphenoidal microsurgery offers long-term, high quality survival for many patients, pituitary surgery has limitations. Even in experienced hands, endocrine and oncological remission is not uniformly achieved. Moreover, tumor recurrence affects a small but significant proportion of patients.

Transsphenoidal surgery remains one of the saf-

Key words: Pituitary surgery, Transsphenoidal surgery, Endoscopy, Neuronavigation, Intraoperative MRI

Address correspondence and requests for reprints to: Panagiotis Nomikos, M.D., Department of Neurosurgery and Gamma Knife Radiosurgery, Hygeia Hospital, 4 Erythrou Stavrou Street, GR-15123 Maroussi, Athens, Greece, TEL: +30-210-6867107, e-mail: nomikosp@otenet.gr

Received 11-11-03, Revised 25-02-04, Accepted 05-03-04 est procedures in contemporary neurosurgical practice. However, complications can occur during or after this procedure, some of which can be associated with significant morbidity and, rarely, also with mortality ${ }^{1}$. Thus, there are still areas in need of improvement $^{2}$. As might be expected, neurosurgeons have tried to develop strategies and new technical tools to improve the surgical outcome. As a result, a variety of important innovations have been introduced during recent years. Among these, the most important and effective have been the use of the endoscope for transsphenoidal surgery, the application of neuronavigation techniques in these skull base procedures and intraoperative imaging, particularly resection control of large tumors by magnetic resonance imaging carried out during the operative procedure.

While some advances are conceptual and others are technical, all are helping to push the limits of pituitary surgery to new frontiers of efficiency and safety. In this paper, the current state of possibilities and limitations of the newly introduced techniques is reviewed with a strong personal bias resulting from the experience of the authors with these techniques. Despite all the technical advances, a proper preoperative workup of the patients, including a complete set of endocrinological, radiological and ophthalmological investigations, an interdisciplinary treatment plan and a precise knowledge of the respective anatomy and the surgeon's experience with the procedure, remain the fundamentals of a successful surgical practice in this area and the basis of satisfaction of the endocrinologist ${ }^{3}$. 


\section{ENDOSCOPIC TRANSSPHENOIDAL SURGERY}

Almost every surgical discipline has been confronted with the increasing demand for minimally invasive procedures. The endoscope was considered an ideal tool for this purpose and was soon incorporated into the armamentarium of the pituitary surgeon ${ }^{4-6}$.

It must be pointed out that even the transsphenoidal approach, carried out in the "classical" way, i.e. with the use of a speculum, has always been considered "minimally invasive"2. However, the surgeon's line of sight is defined by the long and narrow tunnel through which the beam of the microscope reaches the region of interest. An endoscope, added to the classical armamentarium, allows additional insight into the lateral portions of the situs. This supplementation of a traditional approach by a new visualisation tool was called "endoscope-assisted microsurgery" However, the concept of the "minimally invasive" has been redefined in the context of endoscopic approaches to the sella.

Standard ENT-endoscopes allowing a straight view and those with optics of various angles are utilized. These may be used as the sole visualisation tool ${ }^{4,5,6}$. No speculum is used in these procedures. An endoscope is introduced into the nasal cavity down to the vomer. Here a sphenoidotomy is performed either unilaterally or bilaterally. Then both the endoscope and microsurgical instruments are introduced into the sphenoid sinus either through one or both nostrils. Most of the techniques applied in the "classical" transsphenoidal approach are also used in endoscopic surgery. However, instead of the surgeon looking through the ocular of the operating microscope, the structures of the nose, the phenoid sinus and the sella region are displayed on a television monitor. This alters the surgeon's position significantly and requires adaptation to a different operation technique. Alternatively, the advantages of transsphenoidal endoscopy, namely a bright illumination and sophisticated optical system, can be used in addition to performing the "classical" transsphenoidal operation utilizing a speculum and the operating microscope ${ }^{6}$. In both instances, the endoscope allows insight into the lateral portions of the sphenoid sinus and offers the surgeon a superb panoramic visualization of the entire operative field. Knowledge of the anatomical variants that are needed to fully understand endoscopic approaches to the skull base and technical details of endoscopic pitu- itary tumor resection, such as the reconstruction of the sella floor during endoscopic procedures, have been extensively described ${ }^{7,8}$.

In general terms, the anatomical situation encountered during open microsurgical and endoscopic procedures are quite similar (Figure 1). However, the additional anatomical details provided by the endoscope, particularly within the septations of the sphenoid sinus, the protuberances of the carotid arteries in the sphenoid sinus and the anterior confines of the pituitary fossa are mostly displayed in greater detail. Occasionally, we have been able to visualize cavernous sinus involvement and residual lateral tumor. In many cases of cavernous sinus involvement, however, bleeding prevented full use of the virtues of the endoscope and intraoperatively we switched back to open microsurgery.

In summary, convincing evidence that endoscopy improves the outcome or enhances the safety of transsphenoidal surgery of the pituitary gland has not been provided to date. It has been claimed that transsphenoidal endoscopic surgery is a less traumatic approach through the nose. Whether a nasal tampon inserted for 24 hours represents a real disadvantage and discomfort for the patient and if the period of hospitalisation of the patient can actually be reduced cannot be answered. We consider even standard transsphenoidal operations minimally traumatic ${ }^{3}$. This is supported by the fact that the surgery is extremely well tolerated from the standpoint of patient comfort. The direct endonasal approach which we sometimes employ for transsphenoidal surgery, also permits sphenoid sinus access without disruption of the septal

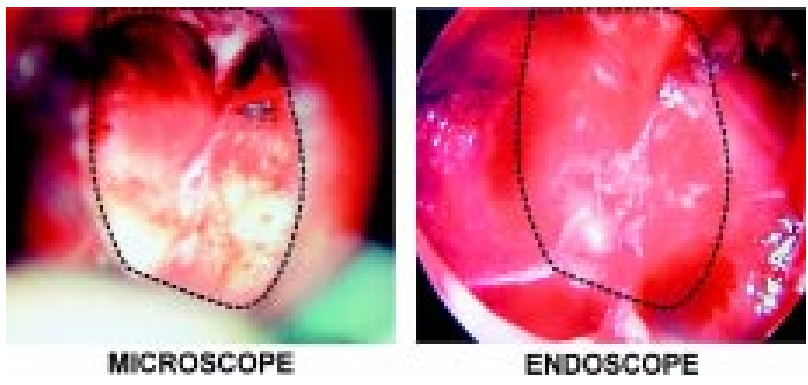

Figure 1. The sellar floor as visualised through the sphenoid sinus with the operating microscope (left) as compared with the endoscope (right). The endoscope allows a more panoramic view, excellently depicting the bone overlying the carotid arteries in the fully pneumatized sphenoid sinus. 
mucosa ${ }^{9}$. The main purpose of postoperative in-hospital surveillance of the patient is that of the potential hormonal imbalance or water- and electrolyte disturbances, since surgery per se is also well tolerated. Since the endoscopic surgeon also interferes with the anterior and posterior pituitary, these problems are not eliminated merely by the use of a new neurosurgical tool. Thus, we do not believe that endoscopical surgery alone can safely reduce hospital stay.

While a huge body of literature ${ }^{10,5}$ underlines the advantages of the use of an endoscope in addition to or instead of standard instruments that are also available in the conventional setting of a "classical" transsphenoidal operation, disadvantages are hardly ever described. A small, specially fashioned mirror introduced through the speculum is a cheap and effective alternative, as it allows indirect visualisation of areas which are initially hidden to the surgeon's eyes. Various endoscopes and a video-camera-system are expensive additional hardware but are now available in almost every hospital. The procedure takes more time and frequently the endoscope manipulation in and out of the nose is not a tissue-friendly behaviour in respect to the nasal mucosa. The optic system is, when introduced close to the real operating site, namely the sphenoid sinus, more prone to opacity by spraying of blood or bone dust than the lens of the microscope, which is placed much more distantly outside the operative field. However, the use of an endoscrub system may overcome this problem.

There is no doubt that endoscopic visualisation greatly helps neurosurgeons to orient themselves with respect to local anatomical landmarks. Thus, endoscopy is certainly a useful technical adjunct to the "classical" armamentarium. Specific surgical tools have been developed so that the surgeons can actually reach what they can see with the new visualisation technique $^{10}$. It seems that further experience will be required to determine the precise role of this new technique.

\section{IMAGE GUIDED SURGERY AND NEURONAVIGATION}

Minimizing tissue trauma is one of the major advances of minimal invasive surgery. However, in many procedures only large exposures allow the identification of a variety of structures, which aid in defining the localisation of the operative field. Thus, with less invasive surgical procedure, fewer anatomical landmarks are available which would aid in proper orientation. Consequently, a need is created for the availability of additional data that would apprise the surgeon as to the exact placement of the instruments in relation to the individual anatomical situation of every patient.

The development of technologies for image guided surgery was one of the major innovations of the past decade. Conceptually, the head is registered in 3-dimensional space to a 3-dimensional dataset acquired with MR or CT. Landmarks to be recognized by the navigation system are attached to the patient's head when the data acquisition takes place. Then, by re-defining these fiducials in the operating theatre, the technology allows the surgeon to navigate to any anatomical landmark or radiologically evident pathological target with precision and safety (Figure 2). Thus, the realistic image of the operative field can be related to the virtual reality of the 3-dimensional data set as it is delivered by the imaging procedure. One of the major advantages of these systems is that selected anatomical landmarks, vascular structures, tumor dimensions and other targets can be manually or automatically segmented and finally superimposed on the surgeon's field of view (Figure 3). Hence, potential operative hazards can be readily recognized by the surgeon while he is carrying out the dissection. There is no clear-cut distinction between intraoperative imaging and navigation, since even the least sophisticated intraoperative imaging usually allows some navigation. Certainly, such systems are not absolutely necessary nor even desirable for routine pituitary surgery. On the other hand, they can be extremely helpful in selected instances. Unusual anatomical variations, prior pituitary surgery and particularly large tumors with unusual extensions are considered indication for image guided surgery.

Vascular anomalies such as kinks and coils of the carotid artery into the sella are the nightmare of the transsphenoidal surgeon and thus represent situations in which even the most experienced surgeon appreciates the advantage of image guidance. Ideally, the segmented vascular structures are projected into the operative field from the very beginning of the procedure. Another potential application is for a poorly pneumatized sphenoid sinus. Navigation may be used 


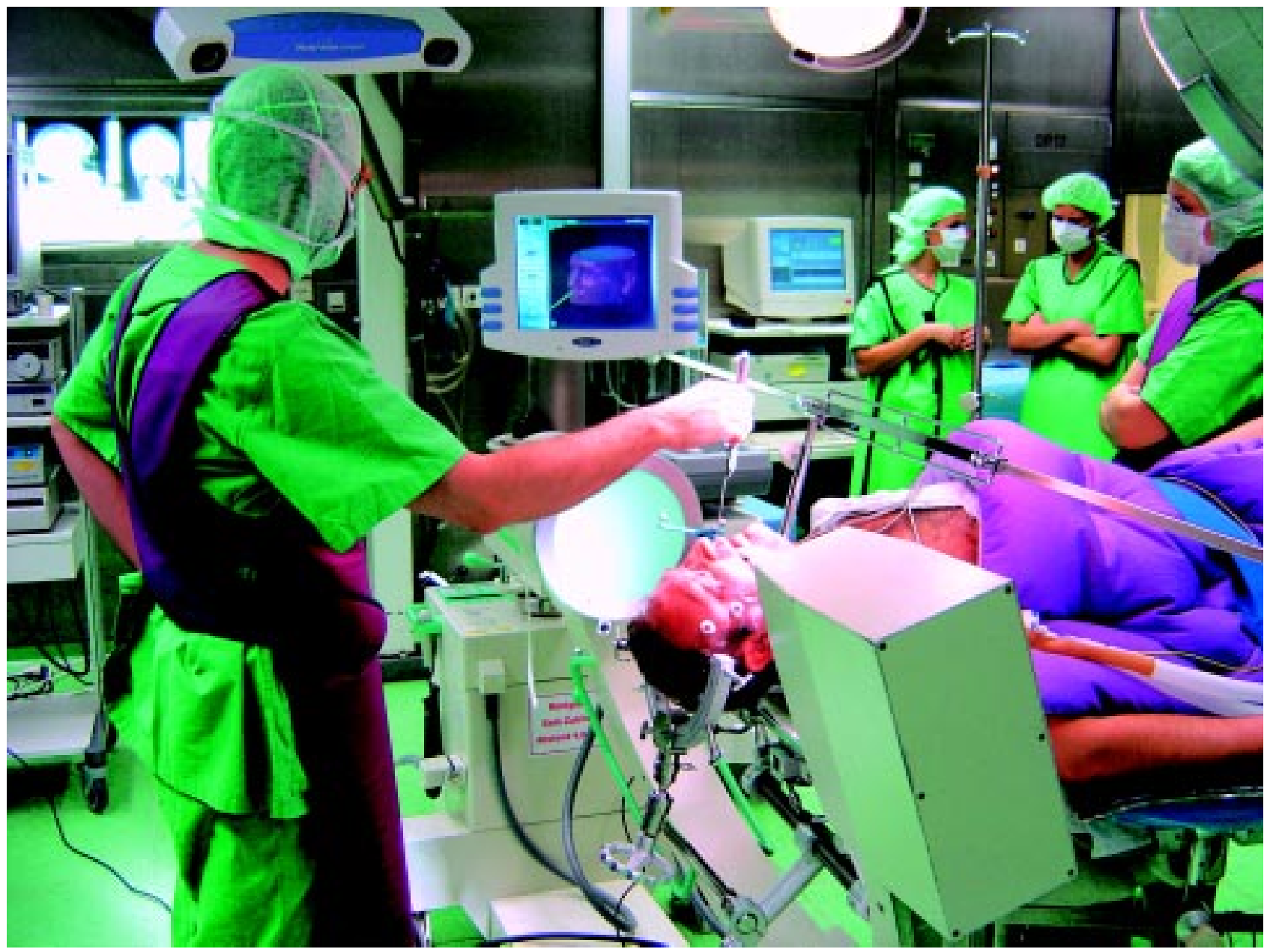

Figure 2. Neuronavigation connects the reality of the surgical field with the virtual world of an MR data set. Both 3-dimensional spaces are related by markers which allow referencing of the neuronavigation system.

for drilling through the osseous portions of the skull base and a safer procedure can result from the use of this technology with a better exposure of the tumor and gland.

A variety of neuronavigation systems have been employed for pituitary surgery with high variations in capability and costs ${ }^{11-13}$. There are pointer-related systems available, which are mainly devised for localisation of a given structure that is encountered during dissection, in the radiological image. These also allow the definition of the ideal trajectory to a given target. The authors believe that microscope-based navigation systems which allow the direct visualisation of segmented structures are even more to the advantage of the surgeon. It is obvious that surgeons less familiar with this procedure will appreciate the use of the systems even during standard procedures. Furthermore, navigation combined with endoscopic surgery adds precision localisation to a minimally invasive procedure ${ }^{14,15}$. The more minimal the exposure, the greater is the need for technology that supports orientation. However, in addition to the relatively high cost of the machinery, the data acquisition for a navigation procedure is comparatively expensive and time-consuming since it requires image data acquisition with fiducials, i.e. the performance of another MR and CT shortly prior to surgery and referencing of the systems with the reality of the patient's head just before the operation itself is commenced. These manoeuvres frequently exceed the duration of the actual surgery itself, particularly when the latter is carried out by experienced staff. 


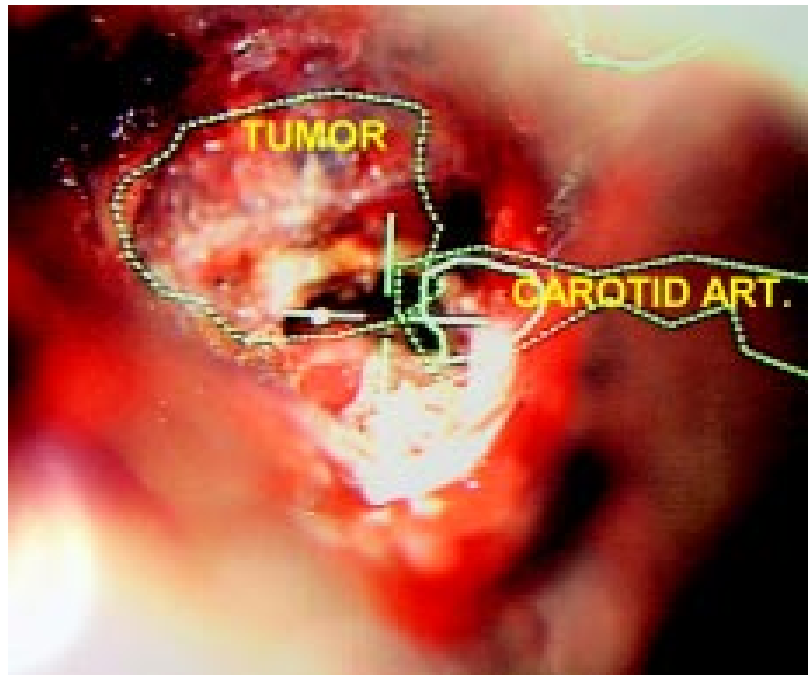

Figure 3. The ideal approach to a pituitary tumor is planned with the navigation system. Relevant structures, such as the carotid arteries and the tumor, may be segmented and specifically displayed in their anatomical relation. These structures (tumor and carotid artery) can be superimposed on the surgical field, on the surface of the sphenoid sinus or on the sella turcica.

\section{INTRAOPERATIVE IMAGING}

The first intraoperative imaging modality utilized during transsphenoidal surgery was $\mathrm{x}$-ray fluorosco$\mathrm{py}^{16}$. However, only bony structures of the skull are visible with the use of an image intensifier. It at least allows an anatomical orientation of the surgeon by visualisation of radioopaque instruments such as steel drills, curettes and forceps in relation to the bony skull base. It is generally also used to direct the nasal speculum towards the sella, thus minimizing the effort of dissection ${ }^{3}$. Experience is available with many procedures, and most transsphenoidal surgeons have used it to date as the standard procedure allowing orientation. The major disadvantage is that it does not directly visualise soft tissues. It is still the most frequently practiced method of both navigation and intraoperative imaging, since the necessary equipment is also required for a variety of other standard neurosurgical operations and thus is available in virtually every neurosurgical department.

In an early attempt to estimate indirectly the extent of tumor removal, intraoperative gas cisternography was used. The intraoperative use of CT-scanning reliably allowed detection of residual tumor for the first time, but did not gain widespread acceptance. Later, ultrasound and doppler-ultrasonography were investigated for intraoperative visualisation of microadenomas in cases of Cushing's disease as well as for the evaluation of the extent of tumor resection in surgery of macroadenomas. The development of low field MR magnets with a so-called "open" configuration, i.e. a horizontal or vertical gap, recently allowed the introduction of MR imaging into the operating room $^{6,17}$. Initial reports demonstrated the feasibility of performing intraoperative MR imaging during transsphenoidal surgery ${ }^{18}$.

Intraoperative imaging may be used for navigation. A titanium needle or a gadolinium filled tube can be used to identify the ideal trajectory to the sella with the image quality strongly depending on data acquisition time. In our experience with the Siemens Magnetom Open scanner, imaging was performed at some stage during the surgical procedure and the major part of the operation was carried out outside the scanner, while in the General Electric (GE) system the surgeon operates within the scanner and thus has on-line imaging throughout the procedure. This explains why we attempted to combine intraoperative imaging and navigation ${ }^{19}$.

It is obvious that complete resection of an intracranial tumor offers an oncological advantage. Morphologically radical tumor resection is a prerequisite for endocrine remission in secreting tumors. Furthermore, in non-functioning tumors, there are no hormonal markers available which would allow an early prognosis of outcome. The intraoperative assessment of the completeness of tumor resection is frequently problematic. The relatively restricted operative field, the existence of recesses of the sella that are beyond the limits of visibility and the need to rely on indirect clues, such as the contour of the diaphragma sellae, frequently complicate assessment of residual tumor, even for the experienced surgeon. Thus, intraoperative visualisation of the completeness of tumor resection by intraoperative MR has been eagerly awaited.

The basic concept of the system used in Erlangen is that transsphenoidal surgery is performed with the patient lying on the tray of the MR scanner ${ }^{6}$. The scanner itself is located in a radiofrequency shielded operating room. The patient's head is placed on a cushion at a distance of $1.5 \mathrm{~m}$ from the centre of the magnet. Standard transsphenoidal surgery is carried out with the patient on the tray slid out as far as possible. For intraoperative measurements an MR-compatible 
titanium speculum is used. The tumor is removed as completely as possible. A piece of bone wax is attached along the remnants of the sellar floor for optimal delineation of the shape of the sella turcica during intraoperative imaging. The table is then moved into the centre of the magnet and imaging is performed ${ }^{6,17}$. Notably, the quality of suprasellar tumor resection is reliably visualized by depiction of decompression of the optic chiasm (Figure 4). We have performed standard transsphenoidal resection in 44 patients with large intra- and suprasellar pituitary adenomas. In these cases intraoperative MR imaging allowed an ultra-early evaluation of tumor resection in $73 \%$ of the cases. Furthermore, a second intraoperative examination in 24 patients with suspected tumor remnants led to an additional resection of tumor tissue in 15 patients $^{6,17}$. Thus, in a substantial number of patients, continued sella exploration permitted a more extensive resection of tumor.
Intraoperative MR imaging undoubtedly offers the option of revising the situs within the same surgical procedure if incomplete tumor resection is suspected. Consequently, the number of procedures during which complete tumor resection is achieved can be improved. Furthermore, additional treatment in cases of incomplete tumor removal can be planned at an early stage, i.e. immediately after surgery. There is no need to wait for delayed postoperative imaging, which is only possible 2-3 months after resolution of artefacts that are not, unfortunately, observable until a few days and even weeks after the procedure. Similarly, intraoperative MR imaging can be used during transcranial surgical procedures for sellar region tumors, such as craniopharyngiomas. We must, however, mention that there are a number of potential artefacts which may create problems with the interpretation of intraoperative images, such as metal artefacts, blood within the sphenoid sinus, etc.
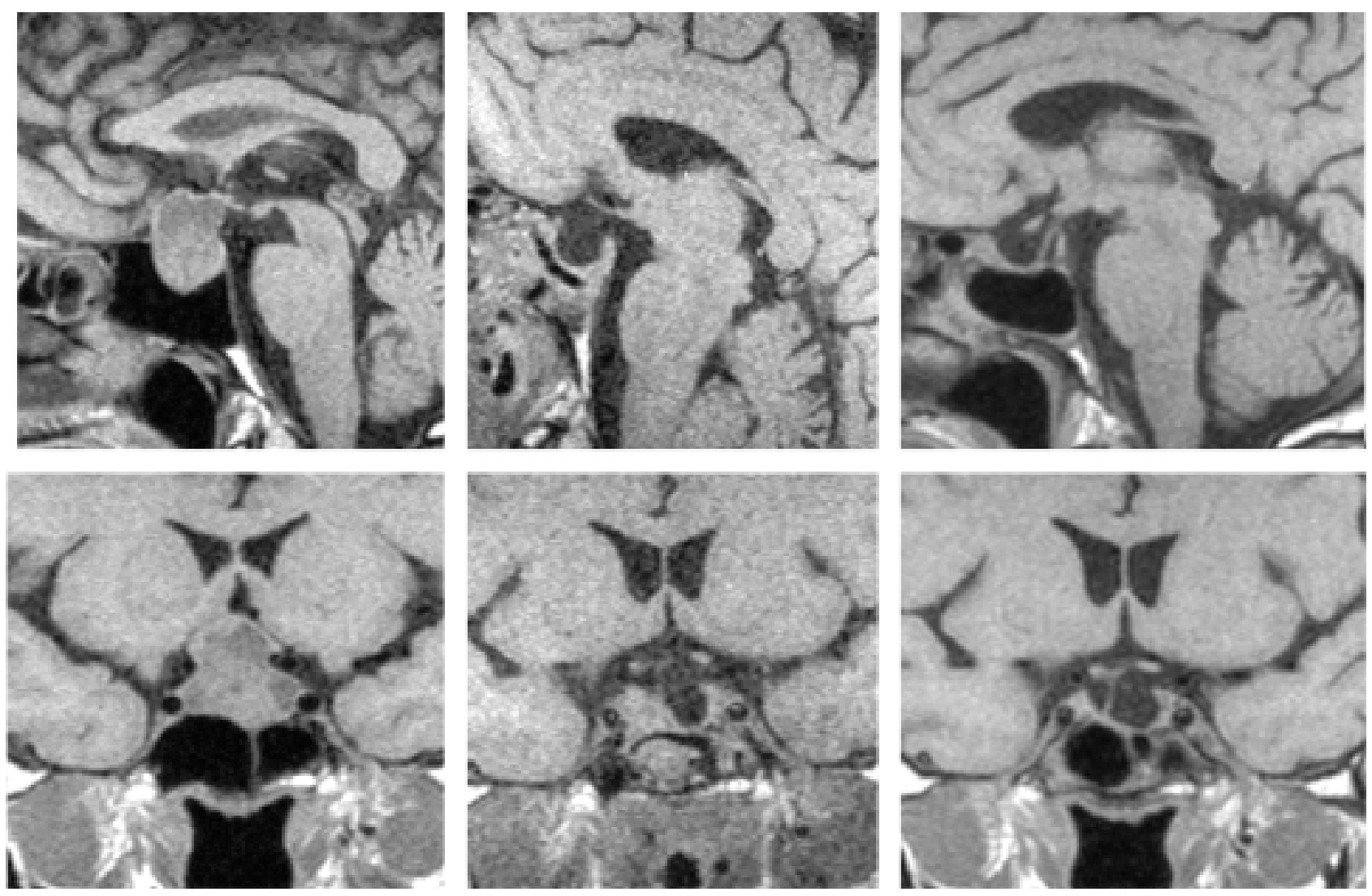

Figure 4. Resection of a large intra- and suprasellar pituitary macroadenoma as documented by intraoperative MR imaging. The upper panel displays the sagittal, the lower the coronal sections, before surgery (left), intraoperatively just after tumor resection (middle), and 3 months after surgery (right). Pre- and postoperative scans were obtained on a conventional 1.5 Tesla magnet, the intraoperative images on the 0.2 Tesla Siemens Magnetom Open scanner. 
Further advances are expected through the introduction of magnets with higher field-strength into the operating theatres. High-field magnets, however, create a variety of additional problems which must be overcome by the development of specific machinery for anaesthesia and operative room equipment, making the installation cost of such systems prohibitive for most hospitals.

\section{CONCLUSIONS}

Since the renaissance of transsphenoidal surgery some forty years ago with the introduction of the operative microscope and the $\mathrm{x}$-ray image intensifier, the transsphenoidal procedure has continued to evolve. A variety of advances have since taken place, which are reflected in technique, instrumentation and strategy. Today, the surgeon has a variety of technical adjuncts available, which serve to maximize the safety and effectiveness of pituitary surgery more than ever before. These developments have, therefore, pushed forward the limits of pituitary surgery. There are still, nevertheless, biological limitations set by the tumor quality, localisation and extension. For more than in the past, appropriate clinical and surgical decision making for each patient is required. The main risk to be avoided in this fascinating new technology is the placing of reliance solely on new technical tools and the exclusion of thought and reasonable appreciation of personal capabilities and limitations. While all the current technical adjuncts together lead to improvements in safety and efficacy of pituitary surgery, they are not by themselves able to replace personal skill, knowledge and experience.

\section{REFERENCES}

1. Fahlbusch R, Buchfelder M 1996 Surgical Complications In: Landolt AM, Vance ML, Reilly PL (eds) Pituitary Adenomas, Churchill Livingstone, New York; pp, 395-408.

2. Fahlbusch R, Thapar K, 1999 New developments in pituitary surgical techniques. Ballière's Clinical Endocrinology and Metabolism 13: 471-484.

3. Buchfelder M, Fahlbusch R, 2002 The "classical" transsphenoidal approach for resection of pituitary tumors. Operative Techniques in Neurosurgery 5: 210-217.

4. Torrens MJ, 1995 Endoscopic neurosurgery. Neurosur- gery Quartely 5: 18-33.

5. Jho HD, Carrau RL, Ko Y, Daly MA, 1997 Endoscopic pituitary surgery: An early experience. Surg Neurol 47: 213-222.

6. Fahlbusch R, Heigel T, Huk W, Steinmeier R 1996 The role of endoscopy and intraoperative MRI in transsphenoidal pituitary surgery In: Werder VK, Fahlbusch R (eds) Pituitary adenomas from basic research to diagnosis and therapy, Excepta Medica Int Congr 1126, Elsevier Amsterdam; pp, 255-268.

7. Nakagawa T, Asada M, Takashima T, Tomiyama K, 2001 Sellar reconstruction after endoscopic transnasal hypophysectomy. Laryngoscope 111: 2077-2081.

8. Nasseri SS, Kasperbauer JL, Strome SE, McCaffrey TV, Atkinson JL, Meyer FB, 2001 Endoscopic transnasal pituitary surgery: Report on 180 cases. Am J Rhinol 15: 281287.

9. Griffith H, Veerappen R, 1984 A direct transnasal approach to the sphenoid sinus. Technical note. J Neurosurg 66: 140-142.

10. Cappabianca P, Alfieri A, Colao A, et al, 2000 Endoscopic endonasal transsphenoidal surgery in recurrent and residual pituitary adenomas: Technical note. Minim Invasive Neurosurg 43: 38-43.

11. Jane JA, Thapar K, Alden TD, Laws ER, 2001 Fluoroscopic frameless stereotaxy for transsphenoidal surgery. Neurosurgery 48: 1302-1308.

12. Kacker A, Komisar A, Huo J, Mangiardi J, 2001 Transsphenoidal surgery utilizing computer-assisted stereotactic guidance. Rhinology 39: 207-210.

13. Sandeman D, Moufid A, 1998 Interactive image-guided pituitary surgery: An experience of 101 procedures. Neurochirurgie 44: 331-338.

14. Kawamata T, Iseki H, Shibasaki T, Hori T, 2002 Endoscopic augmented reality navigation system for endonasal transsphenoidal surgery to treat pituitary tumors: Technical Note. Neurosurgery 50: 1393-1397.

15. Ohhashi G, Kamio, M, Abe T, Otori N, Haruna S, 2002 Endoscopic transnasal approach to the pituitary lesions using a navigation system (Instatrak TM System): Technical Note. Minim Invasive Neurosurg 45: 120-123.

16. Hardy J, Wigser SM, 1965 Transsphenoidal resection of pituitary fossa tumors with televised flouroscopic control. J Neurosurg 23: 612-619.

17. Fahlbusch R, Ganslandt O, Buchfelder M, Schott W, Nimsky C, 2001 Intraoperative magnetic resonance imaging during transsphenoidal surgery. J Neurosurg 95: 381-390.

18. Martin CH, Schwartz R, Jolesz F, Black PMcL, 1999 Transsphenoidal resection of pituitary adenomas in an intraoperative MRI unit. Pituitary 2: 155-162.

19. Nimsky C, Ganslandt O, Kober H, Buchfelder M, Fahlbusch R, 2001 Intraoperative magnetic resonance imaging combined with neuronavigation: a new concept. Neurosurgery 48: 1082-1091. 